\title{
THE POSSIBLE DETECTION OF IRON LINE EMISSION FROM SCO X-1
}

\author{
S. S. HOLT, E. A. BOLDT and P. J. SERLEMITSOS \\ NASA, Goddard Space Flight Center, Greenbelt, Md., U.S.A.
}

\begin{abstract}
A rocket-borne measurement carried out on March 3, 1969 has yielded a net (dead-timecorrected) exposure of $10^{4} \mathrm{~cm}^{2} \mathrm{sec}$ to the differential spectrum of X-radiation from Sco X-1. The data are fully consistent with radiation from an optically thin thermal source, even to the extent that $\mathrm{K}$-emission from high ionization states of iron appears to be present. Such iron emission is consistent with cosmic abundance at the measured temperature.
\end{abstract}

\section{Introduction}

In a recent communication (Holt et al., 1968) we discussed the practical possibility of searching for iron line emission with proportional counters as a positive indication of the thermal nature of discrete X-ray sources. In that communication, we announced our intention of performing an experiment to measure such possible emission from Sco X-1. We report, here, the results of that experiment.

\section{Experiment}

We observed Sco X-1 for $150 \mathrm{sec}$ with essentially the same detector system used in our exposure to the Crab Nebula last year (Boldt, et al., 1969). Almost all of the source exposure is obtained in two nominally identical argon-methane $(10 \%)$ proportional counters with 2-mil beryllium windows. We have chosen to treat the two counters as two independent experiments, partly because the counter responses are not identical and partly because no additional information can be obtained from decreasing the number of degrees of freedom available in the data analysis. The data from one of these counters will be presented here, with the remark that the data from the other are completely consistent with any inferences which we have drawn from these data alone. Furthermore, we nate that the data presented have not been folded back through the detector response; this procedure is necessarily non-unique, and can be particularly misleading if there is a suspicion of discontinuous structure in the input spectrum.

We have started our analysis by fitting the data (with background subtracted) to a single exponential in energy: the standard procedure for analyzing the emission from a suspected thermal source. Figure 1 exhibits this comparison with the best fit exponential normalized to the data between 4 and $15 \mathrm{keV}$ (we pick $4 \mathrm{keV}$ as the lower energy bound to ensure that we are above possible Si and $\mathrm{S}$ edges in the source, and to minimize possible systematic effects arising from minute contaminants in the $\mathrm{Be}$ window). The response of the detector has been exhaustively measured, with the effects of energy dependent efficiency, resolution, escape, internal anode fluorescence 


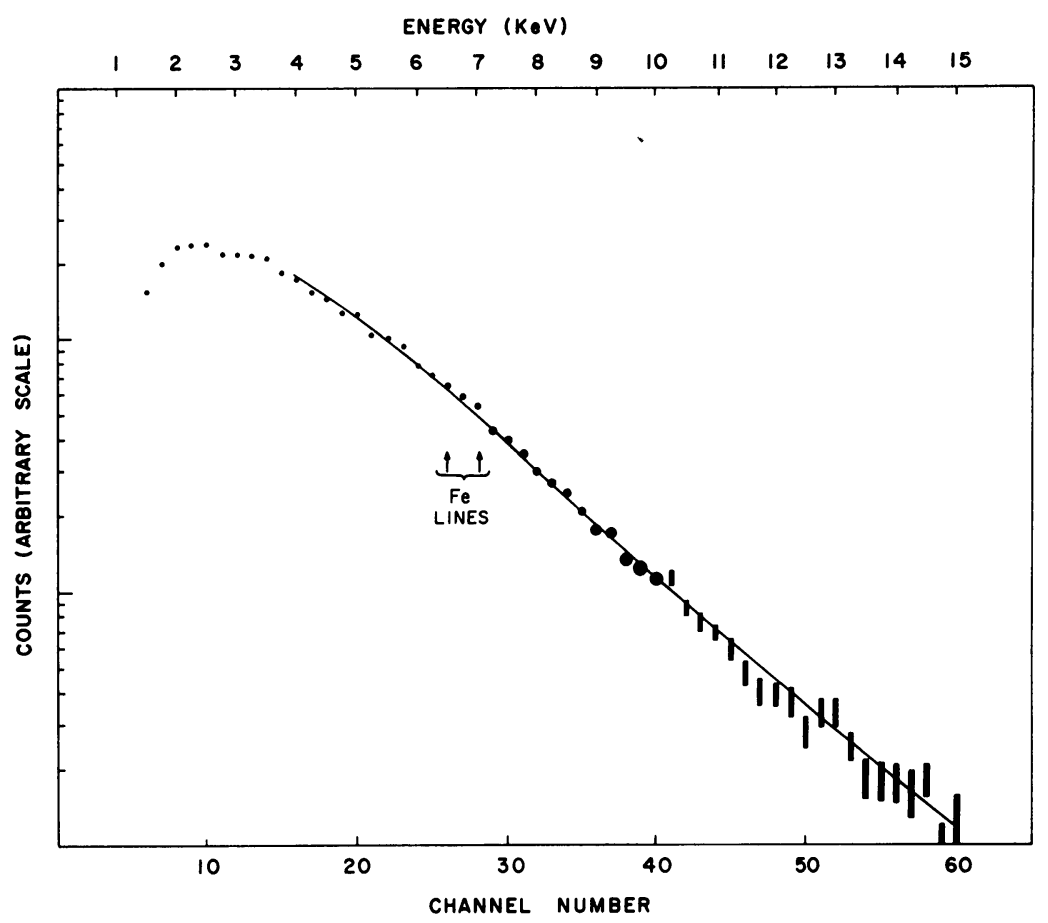

Fig. 1. Comparison of the raw data from one of the two high-exposure counters (with dead-timecompensated background subtracted) with the best fit exponential in differential energy flux folded forward through the detector response. Each point represents $\frac{1}{4} \mathrm{keV}$ channel width, and the statistical error is in the ordinate only. The points have been drawn as circles of radius $1 \sigma$ for observational ease (above $10 \mathrm{keV}$ it became impractical to draw circles). The best fit exponential is normalized to the data in the energy range indicated in the figure: $4-15 \mathrm{keV}$.

and incomplete energy conversion taken into account. The trace in Figure 1 corresponding to the trial spectrum folded forward through the detector response is, therefore, virtually free of systematic error. The fit looks quite good by eye, with the exceptions that there seems to be an excess near the energies corresponding to $\mathrm{K}$ emission from the highest ionization states of iron (as given by Tucker, 1967), a small deviation below the comparison spectrum at lower energies, and an observable deviation below at high energies.

\section{Data Analysis}

Figure 2 indicates the standard quantitative measure of the goodness of fit in terms of $\chi^{2}$. The best fit $\chi^{2}$ is $>70$ at $5.7 \mathrm{keV}$ for 46 degrees of freedom for the simple exponential. $\chi^{2}$ for a pure hydrogen plasma, also shown in the figure, is even worse, and is clearly excluded. The other counter exhibited a minimum $\chi^{2}$ of about the same value at about the same temperature. Since the confidence level at which we obtain a value of $\chi^{2}$ of 70 for 46 degrees of freedom is only $\sim .01$, and we have two such results 
which are statistically independent, the data obviously have too much statistical significance to be fittable to a unit gaunt factor optically-thin isothermal source with no structure.

The most straightforward way to arrive at a consistent input spectrum is to perturb

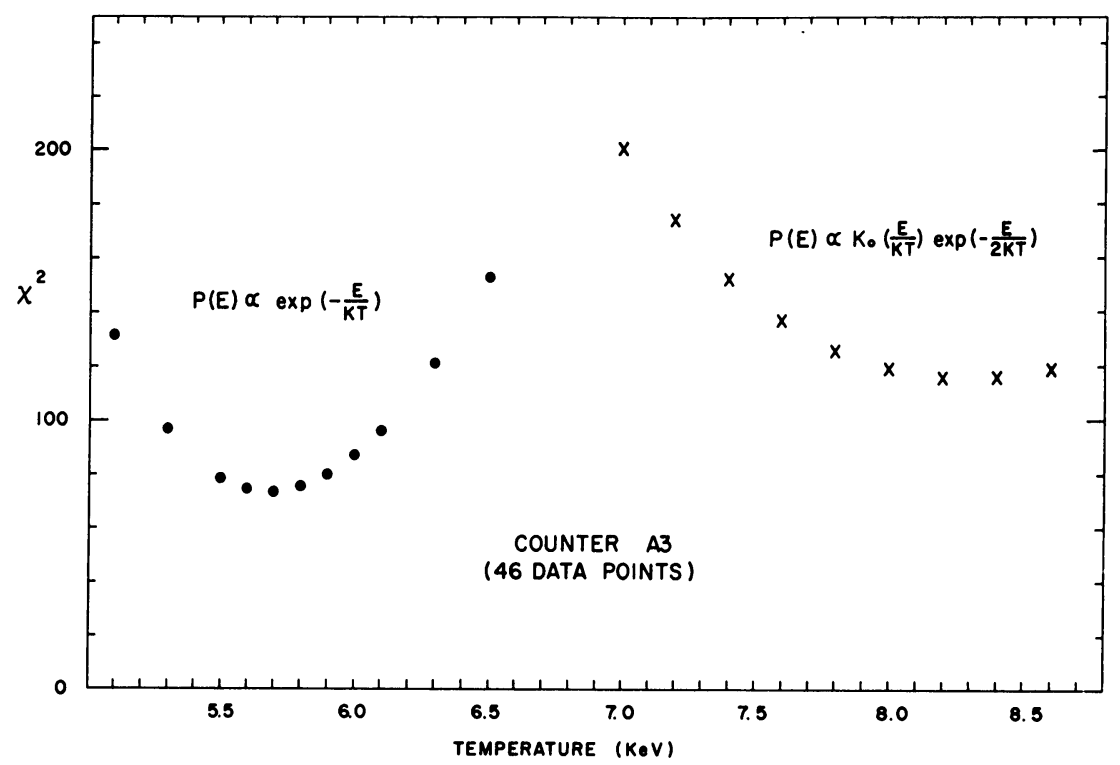

Fig. 2. $\chi^{2}$ for trial exponentials (circles) and trial hydrogenic plasmas (crosses) as a function of temperature for the data shown in Figure 1.

the exponential in a physically reasonable manner. The use of an energy-independent gaunt factor implies the presence of heavy elements, so that the effect of line and recombination radiation on the output spectrum would seem to be the next logical consideration. We have been careful to consider the data above $4 \mathrm{keV}$ only up to this point, because we need not consider the effect of elements with $Z<26$ in a perturbation of the input spectrum if we restrict ourselves to this energy range, since, in fact, the first order effects above $4 \mathrm{keV}$ will be due solely to iron. Figure 3 illustrates, schematically, what the effect of one line and its associated recombination edge would have on a comparison of real data and a pure exponential. The minimum $\chi^{2}$ fit would be the dotted line, at a higher temperature than the actual source temperature, and if one were to plot the percentage deviation between the data points and the trial exponential folded through the detector response, one would expect to see a negative percentage deviation at energies below the line, and another dip at high energies. If the detector resolution is good enough, the intermediate energy structure may be resolvable; the effect of the line and edge emission would be observationally suppressed, however, by the fact that one would be comparing with respect to a higher continuum than actual. 
Such a comparison is displayed in Figure 4. The data are the same data as in Figure 1 , as is the best-fit exponential. At energies below the possible iron lines the deviation is negative, as it is at high energies (past $10 \mathrm{keV}$ the statistical significance of the data points are too poor to plot on this scale). The possibility of iron line emission is

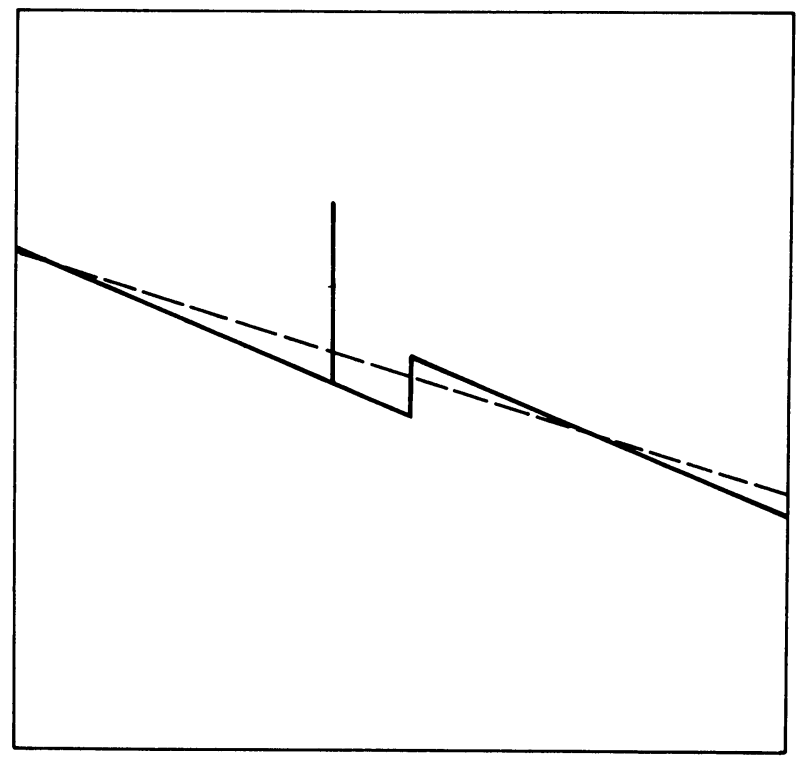

Fig. 3. Schematic representation of the effect of elemental structure in an optically thin isothermal plasma (solid trace) on the determination of the minimum $\chi^{2}$ exponential with which it can be fitted (dotted trace).

intriguing: the $1 \mathrm{keV}$ bin at $6.9 \mathrm{keV}$ is $2.5 \sigma$ above the best-fit exponential, and the $\frac{1}{4} \mathrm{keV}$ points have the shape we would expect to see with our counter resolution. We must remember, also, that this indication of line emission can only be enhanced if we realize that the continuum with which it should be compared is that at lower energies.

\section{Discussion}

It is obvious from Figure 3 that the fit will be better than that obtained with a simple exponential if iron emission is included in the trial spectrum; it remains, however, to determine the amount which is needed for consistency. We have tried fitting Tucker's models II and III (Tucker, 1967), which should be representative of abundances in low and high mass supernovae, to our data. In both cases, the best $\chi^{2}$ was much worse than that for a simple exponential because the iron abundance in these models is much higher than what we observe. The only way that we can get an acceptable value of $\chi^{2}$ is with a smaller iron abundance: in fact, one which must be close to universal.

It is possible to test the hypothesis of universal abundance on the spectrum as a whole. Since, however, the contribution to the X-ray spectrum from each high 
ionization state of every element is small, it is necessary to include them all if one includes any for $Z<26$. We intend to continue our analysis of the lower energy data (our sensitivity extends down to slightly below $2 \mathrm{keV}$ ), but this procedure, if done carefully, will necessarily take a considerable amount of time; this analysis will be complicated, also, by the possibility of optical thickness when we extend the procedure to energies below $4 \mathrm{keV}$. We reiterate, however, that the Sco X-1 spectrum above $4 \mathrm{keV}$ is completely consistent with an isothermal, optically thin source of universal ele-

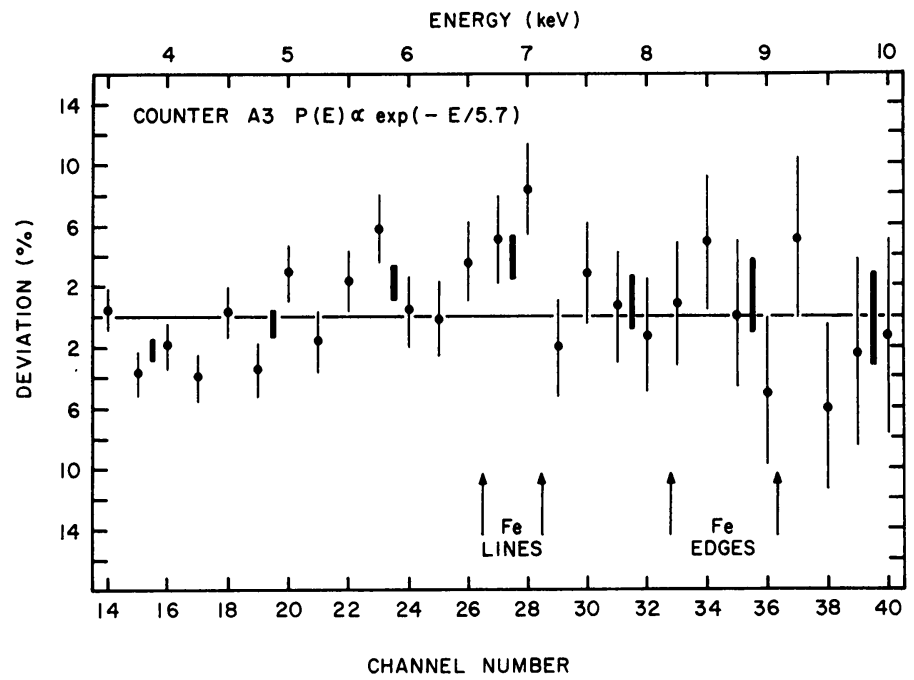

Fig. 4. Percentage deviation of the data from the best fit exponential shown in Figure 1. The points are the same $\frac{1}{4} \mathrm{keV}$ points displayed in Figure 1, while the bars are $1 \mathrm{keV}$ weighted averages of these points centered at the energies shown.

mental abundance. The temperature we measure is essentially identical to that measured by Fritz et al. (1969), and we can exclude distinct overabundances of iron such as demanded by Tucker's models II and III. We can also exclude a pure hydrogenic plasma, and on the basis of a $\chi^{2}$ analysis, conclude that our data can be consistently fit with a source of universal elemental abundance, but not with a pure exponential.

We feel obliged to make a few qualifying comments on the interpretation of our experimental results. The first involves the possibility of a non-thermal (non-collisional) origin for the X-radiation with a spectral shape which masquerades as thermal. We cannot, of course, exclude such a possibility, but we note that the bump is observed at precisely the energy where a thermal source with at least universal abundance of iron would necessarily exhibit such a feature.

Another consideration which would appear to be in order would be the probability that a collisional source would be isothermal. There is no doubt that the celestial object from which the X-ray emission is observed is not isothermal when considered as a whole. The X-ray source, on the other hand, may be well represented by an 
isothermal distribution. Solar active regions (and even large solar X-ray flares) give every indication of being isothermal regions imbedded in a cooler solar corona. The emission from these regions (which have a temperature of only a few times the coronal temperature) completely dominates the observed solar X-ray spectrum. We believe that the objections which may be raised to an isothermal model for the X-ray emitting region on the basis of stellar models which demand temperature gradients are not necessarily valid; the $\mathrm{X}$-ray emitting volume may be virtually isothermal (in analogy with solar active regions) without violating any precepts of stellar model building.

Finally, we address ourselves to the question of iron line emission, per se. Had the input spectrum been characteristic of thermal emission from a supernova remnant (Tucker's models II and III) we could have, unquestionably, made a positive and unambiguous identification of iron line emission (Holt et al., 1968). Since, however, the data indicate that iron line emission, if present, is barely at the level of detectability, we cannot unambiguously conclude that it has been observed. The fact that the inclusion of iron, at approximately the level of universal abundance, is that perturbation of the simple exponential sufficient for complete consistency with our data, would seem to indicate that we have probably observed iron line emission from Sco X-1.

\section{References}

Boldt, E. A., Desai, U. D., and Holt, S. S.: 1969, Astrophys. J. 156, 427.

Fritz, G., Meekins, J. F., Henry, R. C., and Friedman, H.: 1969, Astrophys. J. 156, L33.

Holt, S. S., Boldt, E. A., and Serlemitsos, P. J.: 1968, Astrophys. J. 154, L137.

Tucker, W.: 1967, Astrophys. J. 148, 745. 